\title{
CAMBIOS EN LA FORMACIÓN DEL MAESTRO ESPECIALISTA. UN ENSAYO SOBRE SUS CONSECUENCIAS
}

\section{Autor}

\section{Resumen} Universidad de Las Palmas

Este artículo analiza brevemente las consecuencias potenciales de la reciente propuesta de reforma de los títulos de grado realizada por la Red de Magisterio y la Aneca. Dicha propuesta incluye la supresión de los títulos de especialidad vinculados a materias y una drástica reducción de sus contenidos, junto a varias recomendaciones especialmente críticas que son analizadas por sus consecuencias objetivas y potenciales en diversas áreas del sistema educativo: la movilidad en el EEES, el mercado de trabajo y la promoción académica de los futuros maestros generalistas, la educación de los niños de primaria, la organización de la enseñanza en las escuelas, la eficacia de los estudios científico-técnicos en el ámbito de la educación y los fines de la convergencia europea.

Palabras clave: Formación inicial, Convergencia Europea, Maestro Especialista de Educación Física

\section{Introducción}

Recientemente se ha dado a conocer el Libro Blanco del Título de Grado en Magisterio, hecho que se enmarca en el compromiso de los países europeos por crear un Espacio Europeo de Educación Superior (EEES). Dicho Libro Blanco propone diversas reformas entre ellas la supresión de varios títulos oficiales de maestro especialista, incluido el de educación física, asociado a una reducciónen $2 / 3$ de los contenidos formativos de dichas especialidades (Aneca 2005). Se trata de una reforma importante que afecta directamente al estatus de competencia de los maestros especialistas.

Dicha propuesta de supresión/reducción va unida a dos recomendaciones al Ministerio especialmente críticas, una para que reconozca el $1 / 3$ restante de los contenidos de la especialidad con la capacitación profesional de maestro especialista (op. cit: 201) y la otra recomendación para que no se diseñen títulos de postgrado de las especialidades afectadas (op. cit: 20,200). A la pérdida en el estatus de competencia como consecuencia de la supresión/reducción, habría que añadir su emplazamiento en un callejón sin salida para la formación de un maestro competente en su materia. Por una parte, el pregrado de la especialidad se reduce a un $33 \%$ y el título desaparece. Por otra parte, el postgrado se bloquea y se invita a reconocer como especialistas el $33 \%$ de lo que era antes. La motivación que se ha ofrecido para tal reforma ha sido la convergencia europea, pero sabemos que la convergencia europea y el modelo educativo europeo no amparan tales conclusiones (Serrano, 2004). El Libro Blanco está en la actualidad en fase de tramita- ción bajo la forma administrativa de propuesta o informe no vinculante, para ser discutida y aprobada en el Consejo de Coordinación Universitaria.

De algún modo se puede resumir este proceso como una reforma de los títulos y no una adaptación de los títulos. Es evidente que se ha ido mucho más allá de la convergencia europea y de la idea de una adaptación, trayendo a discusión candente un asunto educativo poco explorado, pero de grandes repercusiones en el sistema educativo como es el empleo de maestros especialistas o generalistas en la educación primaria. La reforma, que no adaptación, del título ha sido abiertamente orientada a la potenciación de la figura del maestro generalista con una mayor formación en ciencias, matemáticas, lengua y enseñanzas plásticas, junto a la eliminación del título de maestro especialista y a una drástica reducción de los contenidos formativos (30 créditos ECTS). Alternativamente se han propuesto que dichos 30 créditos sean elegidos por los alumnos de entre los créditos optativos.

Vista la propuesta de reforma desde un punto de vista cuantitativo, puede afirmarse que casi todas las materias del futuro currículum del maestro tendrán similar cantidad de créditos y estarán en las mismas condiciones de definir un itinerario. Matemáticas o ciencias podrían definir un itinerario que no sería muy diferente en cuanto a peso específico que el de educación física, idiomas o música. En este contexto, la propuesta de itinerarios de especialidad no resulta muy creíble y cuando se analizan las fuentes y los resultados empíricos de los 
trabajos en que se amparan se puede llegar a la conclusión de que la reforma que se propone es esencialmente ideológica, no amparada en la realidad, sino en las concepciones previas que de la educación de los niños o la formación de los maestros tuvo el grupo mayoritario de trabajo. Si el problema fuera prioritariamente ideológico, muchos padres, por ejemplo, podrían negarse a que sus hijos tomaran clases de idioma, música o educación física con maestros que no son especialistas y lo harían con la misma fuerza de razón con la que los expertos han diseñado la reforma. Pero supongo que nadie querría admitir que la reforma ha sido propuesta en base a criterios ideológicos, sino guiada por un enfoque científico-técnico que ha presentado resultados de análisis de fuentes secundarias y encuestas diversas que ya han sido analizadas y discutidas en otro lugar: bien sea en relación a las competencias profesionales (Ceccini, 2004) o bien en relación a la revisión del tópico generalista vs. especialista (Serrano, 2004).

En este artículo se analizará muy brevemente las consecuencias objetivas y potenciales de los variados cambios que se proponen en el Libro Blanco (supresión títulos, reducción de contenidos, bloqueo del post-grado y reconocimiento de la capacitación profesional de especialista). Es una reforma importante porque afecta uno de los pilares que cimientan el hecho educativo, como es el maestro y su formación inicial, con múltiples repercusiones en el sistema educativo. No es racional pensar que este cambio de formación inicial no vaya a tener consecuencias en la educación y el aprendizaje de los niños, porque se trata de un cambio estructural que afectará a muchos elementos del sistema educativo. Para un análisis ordenado de las consecuencias de la reforma acometida se hará un desglose de las áreas potencialmente afectadas: la movilidad en el EES, la inserción laboral y la promoción académica de los futuros egresados, la educación de los niños, la organización de las escuelas, el ajuste a las directrices del EEES y la eficacia de los estudios científico-técnicos orientados a promover reformas en el sistema educativo.

\section{Reducción de la movilidad en el EEES.}

El panorama actual de títulos de magisterio en Europa dista mucho de ser homogéneo. La diversidad es tan amplia que el propio estudio de la Red de Magisterio reconoce la complejidad de ordenar el escenario europeo, reduciendo la realidad de las titulaciones a cuatro estratos de países europeos: a.) solo generalistas, b.) generalistas con itinerarios de especialidad, c.) generalistas con especialidad de pregrado y d.) generalistas con especialidad de postgrado (Carro, 2004). Se trata de un análisis de corte exclusivamente estructuralista que se ha realizado sobre la base de lo que tienen en común los países europeos, dando orden de desahucio a las diferencias transnacionales en la formación de especialistas; de tal modo que la ordenación en cuatro estratos no responde a la realidad por-

que algunos países clasificados como solo generalistas ofertan especialidades concretas de pregrado y postgrado.

Lo que es incontestable es que la práctica totalidad de los países europeos analizados tienen en común una oferta de formación generalista, esto es, los dos títulos de etapa: primariae infantil, pero también es cierto que una buena parte de dichos países ofertan varias especialidadesen sus diversas opciones de pregrado o postgrado y de carácter simultáneo o sucesivo a la formación generalista. Sin embargo, la reforma española de supresión de las especialidades se ha realizado exclusivamente en base al pensamiento atomizado de lo que es común en Europa. Tal es así que la reforma española se basa en un procedimiento de "reducción a lo común". Como las diferencias particulares de las especialidades se suprimieron previamente en el análisis comparativo, el resultado lógico final es una supresión de las especialidades en la adaptación de titulaciones al EES.

De mantenerse los cinco títulos de especialidad de magisterio vinculados a materias, existirían cuanto menos cinco canales de movilidad en el EES, que se añadirían a los dos canales de movilidad que ya de por si suponen las titulaciones comunes de etapa en Europa. La supresión de las especialidades supondrá objetivamente una reducción de las oportunidades de salir a formarse en Europa en materia de educación física, lengua, música o educación especial y sólo se mantendrán como canales abiertos a la movilidad la formación generalista de primaria e infantil.

La supresión de canales de movilidad va en contra de la propia convergencia europea en la que se ampara y en la que se ha formulado claramente la necesidad de diversificar la oferta formativa de pregrado y postgrado para favorecer la movilidad de estudiantes y profesores (declaración de la Sorbona, 1998, párrafo 7: de Praga, 2001, párrafo $2^{\circ}, 7^{\circ}$ y $11^{\circ}$; de Berlín, 2003, párrafo $17^{\circ}$ ). La supresión de la formación de especialistas en el ámbito del magisterio afectará a estudiantes y a profesores. $A$ 
los estudiantes les afectará en las dos direcciones de la movilidad. Los españoles no irán a ningún país europeo para realizar una especialización que no será reconocida académicamente en España y que será recocida profesionalmente en su forma académica de itinerarios. Y a la inversa, los estudiantes europeos no vendrán a España a hacer un itinerario de 30 créditos que no alcanza el mínimo exigido para el reconocimiento de una especialidad en Europa (60 créditos ECTS).

A los profesores les afectará aún más, porque la especialización es una realidad insoslayable del profesorado que dirige la formación inicial universitaria. La movilidad de profesores en Europa se realiza esencialmente en el marco de una especialidad del conocimiento. Si bien la movilidad por motivos de investigación no tiene porque verse afectada, si se verá afectada la movilidad por motivos docentes. En un escenario en el que se persigue aumentar cuantitativamente los flujos de estudiantes y profesores entre los países europeos, atraer estudiantes y profesores de otras partes del mundo y competir en número y calidad con otros destinos de formación superior, la supresión de las especialidades españolas supone en la práctica una supresión de canales o accesos a la movilidad en el ámbito de la formación inicial orientada a la educación de niños de 6 a 12 años.

\section{Estrechamiento del mercado de trabajo.}

Si todas las autoridades educativas europeas, incluido las españolas, decidieran que los niños de primaria solo pueden recibir clases por un maestro de perfil generalista entonces podría encontrarse una base objetiva que justificara la reducción que se propone de los títulos de magisterio a solo dos de carácter generalista (primaria $e$ infantil). Pero tal cuestión no ha sido siquiera evaluada. Los estudios previos realizados por la Red de Magisterio en el proceso de adaptación al EEES no incluyen ninguno que ofrezca información sobre el perfil profesional que se exige en Europa para ejercer la enseñanza primaria. Hay que tener en cuenta que el mercado laboral de los futuros egresados españoles no se circunscribe exclusivamente al mercado español. Se diría como si la propuesta de reforma no contemplara la posibilidad de que los futuros maestros españoles pudieran trabajar en Europa; pero tampoco en España, porque para eso habría que reformar las leyes educativas y suprimir la especialización que en ellas se exige para ejercer la enseñanza en aquellas especialidades que son precisamente las que se han suprimido.

Un estudio de Hall (2000) realizado para el Consejo Escocés de Investigación Educativa, aportó datos sobre el empleo de maestros generalistas y especialistas en la enseñanza primaria europea. Los países que emplean maestros especialistas durante toda la etapa de primaria o en una parte de ella y en una o varias materias, particularmente las que tienen un carácter más instrumental (educación física, idioma, música), conforman el patrón dominante en el mercado laboral de la enseñanza primaria europea. Por otra parte, el mercado laboral español exige por ley maestros especialistas en todos los ciclos de primaria en materias como educación física, música e idioma.
La propuesta de supresión de los títulos de especialista no afronta esta realidad meridianamente clara de la educación formal o intramuros. Pero tampoco la de la realidad extramuros donde se está demandando especialistas de educación física para trabajar con niños de 6 a 12 años en el ámbito de la coordinación de programas deportivos y la recreación física. En principio se pueden objetivar varias consecuencias de la supresión del título.

El incremento de las dificultades para encontrar un empleo en España y en Europa es una de las consecuencias más claras en el ámbito del mercado laboral. La supresión de la especialidad supondrá una restricción de las oportunidades para encontrar un empleo debido a que se cercena la ventaja competitiva que tiene actualmente el título de maestro-especialista, precisamente su doble capacitación de maestro de etapay maestro especialista en una materia. Los maestros ahora solo tendrán una formación generalista y además una amenaza de ver bloqueada su formación de postgrado en la materia específica de su conocimiento: la enseñanza de la educación física en la etapa de primaria. La realidad sin embargo se expresa de otro modo porque la especializaciónes una de las tres cualidades más valoradas por los agentes contratantes en el ámbito de la educación (resultados de la encuesta Cheers, Fundación Bancaja, 2002) y las especialidades suprimidas son las que tienen una mejor inserción laboral (Demométrica, 2004), reflejan mejor las actitudes de los profesionales hacia los perfiles profesionales del maestro (Maldonado, 2004) y las actitudes de los académicos y estudiantes (Loza, 1999, Moreno y Conte, 2001).

Cualquier fuente de datos que se consulte apunta en la dirección de reforzar cuantitativa o cualitativamente las especialidades. Sin embargo se suprimen y se cercena la formación de postgrado. Es un contrasentido que paralelamente se esté promoviendo el reconocimiento de la capacitación profesional. Los itinerarios que se han propuesto previsiblemente condenará a los maestros a trabajar solo en los colegios y eso si el MEC acepta que dichos itinerarios proporcionan capacitación suficiente en relación al currículum de primaria, que no la proporciona. El riego es que solo puedan insertarse laboralmente en los colegios como maestros generalistas y pierdan la posibilidad de insertarse como especialistas en una materia. Las oportunidades de empleo disminuirán en general y el mercado laboral extramuros, con niños de 6 a 12 años, les estará vedado por la competencia que se les hará desde otras titulaciones similares que ofrecen una especialización más consistente.

Además del incremento de las dificultades para que los maestros generalistas encuentren empleo, habría que considerar otras consecuencias derivadas de la reforma de supresión/reducción como es la pérdida de competencias profesionales respecto de los maestros especialistas europeos. Los itinerarios de 30 créditos ECTS nunca podrían ser homologados en Europa como una especialidad académica y en consecuencia tampoco tendrán una capacitación profesional como especialistas en Europa. Solo en España podrían ser reconocidos 30 créditos ECTS en su vertiente de capacitación profesional de especialista y ello si el MEC se aviene a homologarlos. Los maestros españoles con el itinerario de especialidad tendrán menos competencias profesionales como consecuencia del recorte de créditos y del no reconocimiento académico de su título. Los maestros españoles no podrán ir a Eu- 
ropa a ejercer la especialidad y el mercado laboral español puede quedar abierto a los maestros especialistas del resto de Europa si el MEC no se aviene a capacitar profesionalmente los itinerarios de los maestros españoles.

Si tenemos en cuenta los antecedentes históricos en el ámbito de la especialidad de educación física (Fernández Nares, 1993) y de los innumerables cursos de capacitación realizados para cubrir la demanda histórica de especialistas en las escuelas, no descartaría como consecuencia potencial que los licenciados en ciencias de la actividad física tuvieran que recibir en el futuro un curso de adaptación pedagógica para cubrir el potencial vacío en las escuelas de primaria de maestros-especialistas. Hay un riesgo de colisión profesional en una misma etapa educativa de algunas titulaciones de licenciados y maestros que tienen en común compartir una cantidad considerable de créditos académicos y las áreas de conocimiento de su profesorado. De producirse un vacío en el suministro de maestros especialistas al sistema educativo quienes estarian en mejores condiciones de garantizar el cumplimiento íntegro del currículum nacional serían quienes posean una especialidad.

Teniendo en cuenta que los licenciados en CAFD reciben una formación psicopedagógica de base, sería más fácil adaptar a los licenciados a las exigencias de la enseñanza primaria que adaptar a los maestros a la exigencia de la especialidad. Es claro que los licenciados no podrían ejercer como maestros-generalistas pero si como maestros-especialistas. Téngase en cuenta que todos van a tener la misma titulación en el futuro, porque la propuesta de reforma equiparará el título de grado de maestro a un licenciatura de 240 créditos y cuatro años. En términos de la Clasificación Internacional Normalizada de la Educación (UNESCO, 1997) el título de maestro de primaria y el de licenciado en CAFD tendrán la misma clasificación CINE 5 A.

Por último y en este ámbito del mercado laboral sería conveniente traer a discusión la pérdida de una oportunidad histórica para que los maestros ampliaran su escenario profesional al ciclo de la enseñanza secundaria inferior. La carrera, los créditos y el nivel académico se han elevado. Nada impediría que el MEC, legislativa o administrativamente, permitiera que los maestros CINE $5 \mathrm{~A}$ pudieran ejercer la enseñanza en el primer ciclo de ESO porque tienen la misma titulación. Solucionaría muchos problemas que los maestros pudieran enseñar en la etapa inferior de secundaria y creo que sería un elemento importante en la reducción del clima de conflictividad en las aulas en esa etapa de transición que supone pasar del entorno de primaria al entorno de secundaria, donde se pierde la figura del tutor y se podría recuperar con la figura del maestro especialista.

\section{La promoción académica de los maestros generalistas}

Si a quienes hagan un itinerario de 30 créditos ECTS se les capacita como especialistas para el ejercicio de la profesión - aparte de habérseles condicionado la optatividad de los créditos de su carrera a cambio de una especialidad profesional - se les estaría impidiendo el progreso académico de postgrado en la materia específica de su especialización, porque ya no habría necesidad de formar especialistas de postgrado. Ya lo son, pero de pregrado. Quizás por eso la propuesta de reforma contiene dos referencias a la imposibilidad de realizar postgrados en la especialidad de educación física en la enseñanza primaria (Aneca, 2005:20 y 200).

Pero se tendrán que hacer postgrados para que el currículum de primaria no quede desasistido. Dudo mucho que los estudiantes quieran matricularse en un master de especialidad de 60 créditos ECTS cuando con 30 créditos ECTS del itinerario cursado durante la carrera ya tienen reconocida la capacitación. No creo que el altruismo en la formación personal sea un valor actual. El pensamiento racional nos indica que la gente quiere títulos que capaciten para un puesto laboral. Aquellos títulos que solo tengan efectos académicos y no profesionales están llamados a salir fuera del mercado de la educación. Además, la convergencia europea está especialmente llamando a una conciliación de los perfiles académicos con los perfiles profesionales (González y Wagenaar, 2003) y no a un divorcio de los mismos. La desunión especialidad-especialista condicionará la futura promoción académica en la especialidad donde uno ejerce.

Los postgrados del futuro podrán servir para muchas cosas pero no servirán para profundizar en la especialización de la educación física en primaria, cuyo currículum quedará desamparado y ello afectará a los niños. Incluso habrá que ver si los maestros con los nuevos itinerarios son aceptados plenamente como alumnos en aquellos masters específicos de educación física o doctorados que se puedan plantear en el futuro con un nivel CINE 6. Cabe dentro de lo posible que se les exija complementos formativos adicionales como consecuencia de la importante reducción de sus créditos académicos específicos.

\section{La educación de los nitĩos}

La educación de los niños no es responsabilidad exclusiva de la escuela y de los maestros. Sin embargo, la escuela y los maestros son la principal fuente de instrucción y desarrollo de las capacidades de los niños. La eliminación de la figura del maestro especialista reabre las tensiones históricas en el binomio educación-instrucción. Con la reforma se pretende un maestro generalista que sea más competente en la instrucción de las ciencias a costa de sacrificar la formación competente para el desarrollo de otras aptitudes menos intelectualistas como son el conjunto de capacidades físicas, el desarrollo psicomotor o las aptitudes musicales, entre otras. Los estudiantes de magisterio no tendrán crédito académico suficiente para formarse en todas las áreas del currículum nacional de educación física que es la única garantía institucional para un mínimo desarrollo de las conductas y capacidades motoras de los niños. El futuro está llamado a grandes carencias en la aplicación del currículum de primaria. Hay numerosas evidencias de ello.

Las diferencias entre generalistas y especialistas en relación a la enseñanza del currículum nacional ya han sido investigadas en la materia de educación musical comparando las actitudes, la percepción de responsabilidad 
y la auto-confianza en diversas áreas del currículum nacional (Byo, 2000). Este estudio fue concluyente en varias materias. Los especialistas mostraron un mayor grado de familiaridad con los contenidos del currículum, actitudes más positivas hacia todas y cada una de las áreas del currículum, un alto grado de responsabilidad para enseñar todas las áreas del currículum y una mayor percepción de capacidad y entrenamiento para llevar el currículum nacional a la práctica. Por el contrario, los profesores generalistas manifestaron sentirse menos cómodos en todas las áreas del currículum (menos en dos), estaban en desacuerdo con que ellos fueran responsables de la enseñanza de la mayor parte del currículum y tenían una baja percepción de capacidad y entrenamiento para enseñar la mayor parte de las áreas del currículum. En la gestión de los recursos también hubo diferencias. Los especialistas expresaron un acuerdo en que estaban suficientemente equipados para desarrollar $e$ implementar algunas área del currículum y los generalistas creían que tenían pocos o ningún recurso disponible para enseñar todas las áreas del currículum.

El problema subyacente reside en la queja reiterada planteada por los maestros generalistas acerca de la creciente saturación de contenidos en los currículos nacionales (Campbell, 1992; Williams, 1990; Hounshell \& Swartz, 1987) y la necesidad consiguiente de "descargar" a los maestros generalistas con distintas figuras de especialistas o semiespecialistas (Alexander et al., 1992; OFSTED, 1993, 1994, 1997) y así evitar un ciclo de descuido en la enseñanza de las materias afectadas (Duck, 1990, Williams, 1990, Abell, 1990). Por otra parte se sabe que la falta de autoconfianza y las limitaciones en el conocimiento del contenido de la materia son características que correlacionan con los maestros generalistas, particularmente en la enseñanza de las materias más instrumentales (Bennet y Carré, 1993; Duck, 1992). También se ha probado la relación entre el número y calidad de las experiencias educativas de los alumnos con el conocimiento del contenido de la materia (Barry, 1992; Cassidy, 1990; Mullins, 1993). La figura del maestro generalista ha sido muy cuestionada como un elemento que beneficie la enseñanza y el aprendizaje.

El conocimiento educativo actual va orientado a que no todas las materias del currículo de primaria son igual

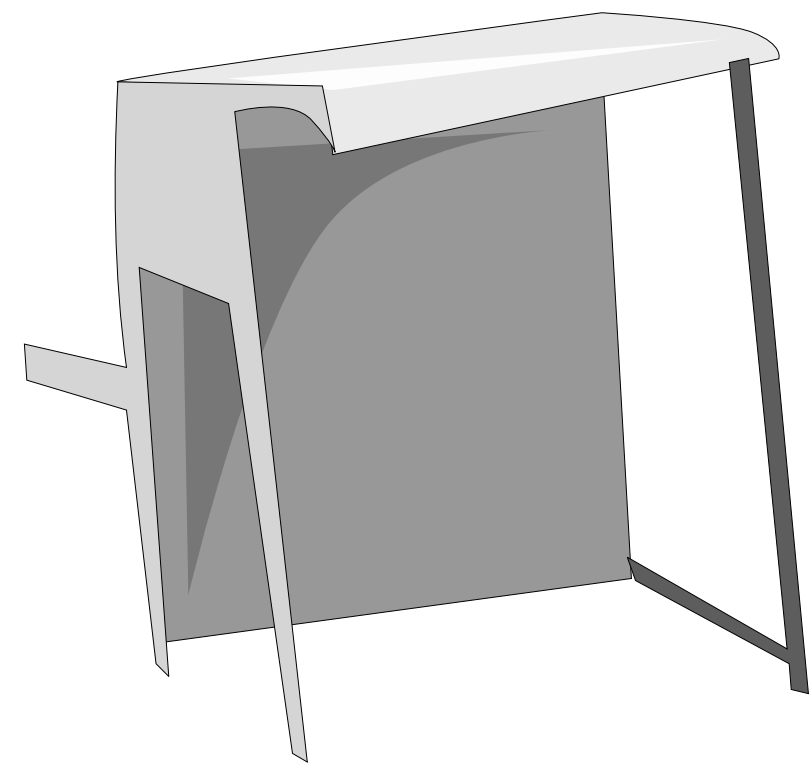

de importantes en el desarrollo de los niños, porque estos atraviesan periodos con una sensibilidad diferenciada a la estimulación y el aprendizaje. La educación física y motriz, la enseñanza de los idiomas y de la música son más oportunas de estimular en la infancia y la niñez, y por ello el legislador confió estas materias al empleo de maestros-especialistas. La supresión de los títulos y la disminución de sus contenidos formativos afectarán inevitablemente a la competencia del maestro y a su conocimiento del contenido de la materia que es el factor más limitante de la calidad de la enseñanza (Cassidy, 1990; Mullins, 1993; Reimer, 1993; Gess-Newsome, 1999).

En el caso de la educación física, la supresión de la especialidad de primaria se hace en un momento especialmente crítico relacionado con las pandemias de inactividad, obesidad, diabetes y osteoporosis que a menudo son caracterizadas como problemas pediátricos de consecuencias geriátricas o en la edad adulta. El problema es creciente y de tal magnitud que en algunos países ya se han incluido objetivos nacionales de salud pública relacionados con la actividad física en la escuela. En el caso de Estados Unidos, por ejemplo, el plan nacional de salud "Healthy People 2000 objectives" (US Department of HHS, 1996) ha incluido como objetivos nacionales incrementar hasta al menos el $50 \%$ la proporción de niños que participan en programas de educación física escolar, además de otro objetivo directamente relacionado con la didáctica de su enseñanza como es incrementar hasta al menos el $50 \%$ la proporción de tiempo, en las clases de educación física, en que los niños son físicamente activos.

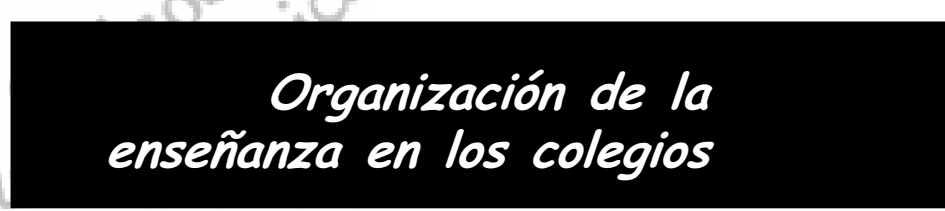

La propuesta de reforma de supresión/reducción se ha hecho con el pleno conocimiento de que las leyes educativas (LOGSE o LOCE) exigen el empleo de maestros especialistas y aún asi se propone la supresión académica de esta figura. La propuesta de reforma no respeta las leyes aprobadas por el Congreso Nacional y fuerza una situación comprometida como es tener que reformar las grandes leyes educativas para forzar su ajuste al Libro Blanco de los títulos de magisterio o bien tener que otorgar la capacitación profesional como especialista a quienes hagan los 30 créditos optativos de un itinerario.

Cabe dentro de lo posible que los itinerarios no sean homologados con la capacitación profesional de especialistas por desajustes legales con el recientemente aprobado real decreto de los títulos de grado que prohíbe expresamente el reconocimiento de una especialidad académica dentro de un título generalista. Si esto ocurre se producirá inevitablemente un colapso progresivo de maestros especialistas en las escuelas a causa del desabastecimiento en la formación de egresados. En este caso el escenario futuro está lleno de incertidumbres y cuanto menos la reforma propuesta de supresión/reducción en vez de solucionar problemas en el sistema educativo, los agravaría, constituyéndose en si misma como una fuente de problemas innecesarios en la organización de las enseñanzas en los colegios de primaria. 


\section{La eficacia de los estudios científico-técnicos en materia de educación}

La propuesta de supresión/ reducción se amparó básicamente en dos estudios empírico-analíticos realizados por la Red de Magisterio: "Los títulos de grado en Europa"y el "Cuestionario de opinión sobre los perfiles profesionales de los maestros" cuyas conclusiones son en cierto modo sorprendentes, porque no concuerdan con los resultados y son contradictorias entre sí. Concretamente, el segundo estudio citado, que fue el más decisivo, aportó cuatro conclusiones contrarias a los fines por los que se hizo el estudio, que era precisamente aportar resultados concluyentes sobre la actitud de los maestros egresados y otros agentes educativos hacia las especialidades LOGSE y LOCE. Este estudio no fue capaz de determinar tal objetivo investigador. La decisión final de cuál de las cuatro conclusiones era la más verdadera se realizó mediante votación, que es esencialmente un acto político, y salió ganadora la consabida conclusión de solo dos títulos de grado de carácter generalista (Aneca, 2005:79). Es obvio que para este desenlace mediante votación no hacía falta la realización de estudios previos. De similar modo ocurrió con el primer estudio citado, donde pese a concluir que una mayoría de países ofrecen formación de especialidades, se termina proponiendo que la formación especializada se suprima y se subordine a la formación en ciencias, matemáticas y lengua. Que es precisamente el núcleo duro de la propuesta de reforma de las titulaciones.

Parece meridianamente claro que la ANECA, en el marco de su proceso de evaluación de las propuestas de adaptación de los títulos, no incluye dentro de su protocolo una revisión de los cuestionarios, escalas, estadística, resultados y conclusiones de los estudios previos que amparan tales propuestas. Se hicieron varios estudios además de los anteriormente indicados: "La inserción laboral de los titulados de magisterio durante el ultimo quinquenio (Demométrica, 2004), "La oferta pública de empleo (Ruiz, 2004)" y la "Oferta, demanda y matrícula de los títulos de maestro" que no han sido tenidos en cuenta para nada. El conjunto de estos estudios era claramente a favor de mantener las especialidades e incluso reforzarlas. Igualmente otros estudios necesarios, como el "Empleo de maestros especialistas en la enseñanza primaria europea" no han sido siquiera abordados y pese a que son mayoría los países europeos que emplean maestros especialistas en diversas materias y ciclos de la enseñanza primaria como antes se señaló.

La reflexión que inevitablemente surge es para qué sirven los estudios empírico-analíticos cuando las decisiones sobre sus conclusiones se van a tomar a un nivel ideológico. El hecho de los resultados científicos se subordinen al ejercicio de una mayoría o de la ideología dominante va en detrimento de la credibilidad y eficacia de la ciencia y la razón. Si los estudios se elaboran para someterlos después a la consideración mayoritaria de un grupo de representantes para ver cual de las diversas conclusiones es la más verdadera no creo que estemos avanzando en la dirección europea adecuada porque la ciencia y la razón estarían siendo suplantadas por las mayorías cualificadas.

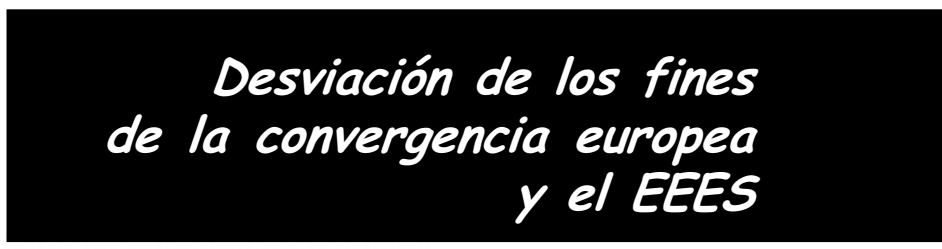

La propuesta de la adaptación de las titulaciones en magisterio al EEES no se adapta a las numerosas recomendaciones presentes en el proceso de convergencia europea. La reducción a lo que es común (dos títulos de etapa) choca abiertamente con los numerosos pronunciamientos sobre la defensa y promoción de la diversidad de estudios y módulos como mecanismos para promover la movilidad (declaración de la Sorbona, 1998, párrafo $7^{\circ}$. de Praga, 2001, párrafo $2^{\circ}, 7^{\circ}$ y $11^{\circ}$; de Berlín, 2003, párrafo $17^{\circ}$ ), además de no reflejar la realidad de las titulaciones europeas. La consecuencia es una incongruencia de la reforma propuesta en relación al proceso de convergencia europea.

Otra de las consecuencias de la reforma de supresión es la desvirtuación de un objetivo de primera magnitud de la convergencia europea como es la necesidad de unir los títulos y los perfiles profesionales. El proyecto Tuning (González y Wagenaar, 2003), de máximo nivel en la Comisión Europea, ha dejado claro la necesidad de que la construcción del EES refuerce la integración de la dimensión académica de los títulos con la dimensión profesional de los perfiles. Se ha hecho un extraordinario esfuerzo por encontrar el elemento catalizador de dicha unión o integración, precisamente las competencias profesionales. Sin embargo, la supresión del título de maestro-especialista y la solicitud que al mismo tiempo se hace para que se reconozcan sus competencias profesionales suponen una clara desviación del proceso de convergencia europea y lejos de reforzar la dimensión académica con la profesional, ahondará en su crisis en un momento en que se perseguía precisamente lo contrario.

\section{Referencias Bibliograficas}

ANECA (2005). Libro Blanco. Título de Grado en Magisterio. Vol. 1. Agencia Nacional de Evaluación de la Calidad y Acreditación: Madrid.

Abell, S.K. (1990). A Case for the Elementary Science Specialist. School Science and Mathematics, 90 (4), 291301.

Alexander, R., Rose, J. and Woodhead, C. (1992) Curriculum Organisation and Classroom Practice in Primary Schools. London: Department of Education and Science.

Barry, N.H. (1992). Music and education in the elementary music methods class. Journal of Music Teacher Education, 2 (1), 16-23.

Bennett, N. and Carré, C. (eds.) (1993). Learning to Teach. London: Routledge. 
Byo, S.J. (2000). Classroom Teacher's and Music Especialist' Perceived Ability to Implement the National Standards for Music Education. Journal Research in Music Education, 47 (2), 111-123.

Campbell, J. (1992). Class teaching: the nightmare at Key Stage 2. Junior Education, 22(1), 16-17.

Carro, L (2004). Los grados de magisterio en los 25 países de la Unión Europea. Informe provisional elaborado en el proceso de "Adecuación de las titulaciones de maestro al EEES". Red de Magisterio. www.ub.es/ffpro/ licenciatura/aneca_maestros.

Cassidy, J.W. (1990). The effect of intensity training on preservice teachers' instruction accuracy and delivery. Journal of Research in Music Education, 38 (3), 164 174.

Cecini, J.A. (2004). Los contenidos de la formación inicial ¿Pedagógicos o de conocimiento? Actas del XXII Congreso Nacional de Educación Física. Universidad de A Coruña.

Comunicado de Berlin (2003). Realising the European Higher Education Area. Communiqué of the Conference of Ministers responsible for Higher Education. Berlín, 19 de Septiembre. www.bologna-berlin2003.de/pdf/ Prague_communiquTheta.pdf

Comunicado de Praga (2001). Towards the European Higher Education Area. Communique of the meeting of European Ministers in charge of Higher Education. Prague, 19 de Mayo. www.bologna-berlin2003.de/pdf/ Prague_communiquTheta.pdf

Declaración de la Sorbona (1998). Declaración conjunta para la armonización del diseño del Sistema de Educación Superior Europeo. París, 25 de Mayo. www.universia.es/contenidos/universidades/documentos.

Declararación de Bolonia (1999). El Espacio Europeo de la Enseñanza Superior. Declaración conjunta de los ministros europeos de educación. Bolonia, 19 de Junio. www.aneca.es/modal_eval/docs/declaracion_bolonia.pdf

Demométrica (2004). Informe sobre el cuestionario de inserción laboral de los maestros. Informe elaborado en el proceso de "Adecuación de las titulaciones de maestro al EEES". Red de Magisterio.

Duck, G. (1990) The Arts in Primary School and the Preparation of Teachers to Teach the Arts: results and implications of a research study. South Pacific Journal of Teacher Education. 18 (2). 119-126.

Fernández Nares, S. (1993). La Educación Física en el sistema educativo español: la formación del profesorado. Universidad de Granada: Granada.

Fundación Bancaja (2002). La situación laboral de los graduados españoles. Capital Humano, n² 21, 3-7.

Gess-Newsome, J. (1999). Delivery models for elementary science instruction: A call for research. Electronic Journal of Science Education, 3(3), 1-8.
González, J. and Wagenaar, R. (eds) (2003). Tuning Educational Structures in Europe. Informe Final. Fase Uno. Universidad de Deusto: Bilbao.

Hall, J. (2000). Initial Teacher Education: Specialists and Generalists. Scottish Council for Research in Education. www.scre.ac.uk/scot-research

Hounshell, P.B. \& Swartz, C.E. (1987). Elementary Science Specialists? Definitely!. Science and Children, 24(4), 20-21, 157.

Loza Olave, E. (1999). En torno al currículo en la formación de los maestros especialistas en educación física. Bordon, 51 (1), 39-50.

Maldonado Rico, A. (2004). Informe final sobre el cuestionario de opinión sobre los perfiles profesionales de los maestros. Informe elaborado en el proceso de "Adecuación de las titulaciones de maestro al EEES". Red de Magisterio. www.ub.es/ffpro/licenciatura/ aneca_maestros.

Moreno, J.A. y Conte, L. (2001). La valoración de la especialidad de educación física y capacitación docente a través del alumnado. Revista Electrónica Interuniversitaria de Formación del Profesorado, 4 (1). www.aufop.org/publica/reifp/01v4n1.asp

OFSTED (1993) Curriculum Organisation and Classroom Practice in Primary Schools: a follow-up report. London: Department for Education.

OFSTED (1994) Primary Matters. London: HMSO.

OFSTED (1997) Using subject specialists to promote high standards at Key Stage 2: an illustrative survey. London: OFSTED

Reimer, B. (1993). Avoiding extremes of theory and practice in music teacher education. Journal of Music Teacher Education, 3 (1), 12-21.

Ruiz Juan, F. (2004). Oferta de empleo público. Oposiciones a magisterio 1991-2003. Informe elaborado en el proceso de "Adecuación de las titulaciones de maestro al EEES". Red de Magisterio. www.ub.es/ffpro/licenciatura/aneca_maestros.

Serrano (2004). La educación física en el marco de la formación inicial de un profesor generalista o especialista. Actas del XXII Congreso Nacional de Educación Física. Universidad de A Coruña.

Unesco (1997). Clasificación Internacional Normalizada de la Educación. CINE, 1997. Unesco. http:// unesdoc.unesco.org/images/0010/001089/108954s.pdf

Williams, D.H. (1990). Making a case for the science specialist. Science and Children, 27 (4), 30-32.

US Department of HHS. (1996). Physical Activity and Health: A Report of the Surgeon General. Atlanta, GA: US Departament of Health and Human Services, Centers for Disease Control and Prevention, National Center for Chronic Disease Prevention and Health Promotion. 